\title{
Relativistic Quantum Mechanics Through Frame-Dependent Constructions
}

\author{
Jeffrey A. Barrett \\ 28 July 2004
}

\begin{abstract}
This paper is concerned with the possibility and nature of relativistic hidden-variable formulations of quantum mechanics. Both ad hoc teleological constructions of spacetime maps and frame-dependent constructions of spacetime maps are considered. While frame-dependent constructions are clearly preferable, they provide neither mechanical nor causal explanations for local quantum events. Rather, the hiddenvariable dynamics used in such constructions is just a rule that helps to characterize the set of all possible spacetime maps. But while having neither mechanical nor causal explanations of the values of quantummechanical measurement records is a significant cost, it may simply prove too much to ask for such explanations in relativistic quantum mechanics.[1]
\end{abstract}

\section{Teleological Constructions}

It is difficult to find a satisfactory formulation of relativistic quantum mechanics. More specifically, it is difficult to provide a dynamical account of the process of measurement and the production of determinate measurement records that is compatible with the constraints of relativity (Barrett 2003). This paper concerns the most direct way of providing a hidden-variable formulation of quantum mechanics that is compatible with relativity. The strategy guarantees determinate measurement records but ultimately forfeits any 
mechanical or causal explanation of the production of such records or of any other physical process.

One might naturally suppose that a formulation of quantum mechanics is compatible with relativity if it can be given without reference to any preferred inertial frame. And, in the context of special relativity, one might suppose it to be sufficient that the dynamics assign a unique local quantum-mechanical state to all regions in Minkowski spacetime. But if this is all one requires, then it is easy, perhaps too easy, to get a formulation of quantum mechanics that is compatible with relativity.

Bloch (1967) provided an early discussion of the difficulties one faces in reconciling quantum mechanics and relativity. He understood the problem as one of finding a collapse formulation of quantum mechanics that (i) was compatible with the constraints of special relativity and (ii) would explain the determinate measurement records generated by particle detection experiments. Using particle counters as quantum measuring devices, Bloch explained how one might get a weak sort of compatibility with relativity by supposing collapses to occur along the backward light cones of measurement interactions.[2]

Bloch concluded his discussion of relativistic quantum mechanics by describing how one could, if one pleased, define a "teleological wave function" by simply stipulating the value of the wave function so that it corresponds to whether or not each particle counter is triggered at each particular location in spacetime where a measurement is made $(1967,156)$. That is, one might construct an empirically adequate spacetime map of the quantum-mechanical state by stipulating that the local value of the wave function in each region of Minkowski spacetime is an eigenstate of the recording variable wherever and whenever there is in fact a measurement record. One might then complete the spacetime map by filling in local values for the wave function in all other regions subject to the constraints imposed by the values in those regions where there is a determinate record. There are several ways one might do this. If one used the standard unitary dynamics to fill in the values outside the determinate-record regions (insofar as possible), then the resultant spacetime map might look as if collapses of the wave function had generated the determinate measurement records. Indeed, such a complete spacetime map might be constructed from Bloch's backward light-cone collapse prescription above if one knew the result of each measurement. But there is a sense in which it does not really matter how one completes the spacetime map since 
one already has the right determinate measurement records by stipulation. In any case, a teleological spacetime map constructed from observed measurement records would clearly be both empirically adequate (by stipulation) and perfectly compatible with relativity (insofar as it is just a map of local quantum-mechanical states in Minkowski spacetime).

A closely related procedure would be to stipulate the values of the determinate measurement records in only some privileged spacetime region (rather than the values of all measurement records), then to use these local records together with the unitary dynamics to construct a fictional wave function for all spacetime regions that represents the conditional probabilities of various possible records in those regions given the stipulated records in the privileged region. A wave function so constructed would be fictional outside the privileged region since, assuming the standand eigenvalue-eigenstate link (more specifically, without the introduction of hidden-variables), the actual wave function would be an eigenstate of whatever measurement records are in fact obtained outside the privileged region and would consquently assign probability one to each actual measurement record and probability zero to all other possible records. Since it does not determine the values of the determinate physical records there and consequently cannot be taken to provide a complete and accurate representation of the state outside the privileged region, one might naturally conclude that such a fictional wave function represents possible epistemic states of observers who only know the actual measurement records in the privileged region. In any case, given the standard eigenvalue-eigenstate link, it is the teleological state map, which can only be constructed here after one knows the value of all measurement records, not a fictional state map representing conditional probabilities, that can be taken to represent the complete quantum-mechanical state in each region of spacetime.[3]

Concerning the construction of such ad hoc teleological spacetime maps, however, Bloch concluded that "such a procedure appears to have little to recommend it" $(1967,156)$. While one cannot help but agree with this conclusion, it is also unsurprising to find such teleological constructions throughout the literature on relativistic quantum mechanics given the difficulties inherent in finding a dynamical account of the quantum measurement process that is compatible with relativity.

Hellwig and Kraus (1970) have proposed adopting precisely the ad hoc procedure that Bloch found objectionable in order to extend Bloch's dis- 
cussion of particle-detection records to the construction of spacetime maps for quantum field variables. The procedure here is simply to stipulate the corresponding local value of the field variables in those spacetime regions where there are in fact determinate measurement records, then to fill in the quantum-mechanical field state map in other regions using the standard unitary dynamics. Hellwig and Kraus' concrete proposal for how to complete the spacetime map amounts to an application of the collapse dynamics along the backward light cone of each measurement event in spacetime and an application of the standard unitary quantum dynamics everywhere else (1970, $567)$.

While such spacetime maps can clearly be taken to be empirically adequate models of any collection of actual determinate measurement records and while there is a sense in which they are also clearly compatible with the constraints imposed by special relativity, they are nevertheless blatantly ad hoc. Such constructions guarantee empirical adequacy by stipulation. While such a spacetime map may predict and explain our empirical results in the sense of logically entailing the determinate records we in fact find, the predictions are ad hoc and the explanations are the most impoverished imaginable.

The salient question is whether we can do any better than such ad hoc constructions of quantum-mechanical spacetime maps. I think we can. In particular, I will argue that frame-dependent constructions of hidden-variable spacetime maps are clearly preferable. While I take frame-dependent hiddenvariable constructions to be preferable, they also limit the sort of explanations available in relativistic quantum mechanics. I will use both basic and generalized Bohmian mechanics to illustrate frame-dependent hidden-variable constructions. Then I will discuss the relative virtues and vices of such constructions.

\section{Frame-Dependent Constructions}

As the most popular hidden-variable formulation of quantum mechanics, Bohm's theory (1952) provides a convenient context for discussing framedependent constructions (This description of Bohmian mechanics follows Bell 1981 and 1982).

In basic Bohmian mechanics, a complete physical description consists of the standard quantum-mechanical state $\psi$ together with a specification of the 
always-determinate position $Q$ of each particle. It is the determinate particle configurations relative to the wave function that are supposed to explain our determinate measurement records in this theory.

According to basic Bohmian mechanics, the standard quantum-mechanical state, always evolves in the standard deterministic unitary way. In the simplest nonrelativistic case, this evolution is described by the time-dependent Schrödinger equation

$$
i \hbar \frac{\partial \psi}{\partial t}=\hat{H} \psi
$$

where $\hat{H}$ is the Hamiltonian of the system.

The determinate particle configuration $Q$ also evolves in a deterministic way. For an $N$ particle system, the particle configuration can be thought of as being pushed around in $3 N$-dimensional configuration space by the flow of the norm-squared of the wave function (the probability current) just as a massless particle would be pushed by a compressible fluid. More specifically, the motion of the particles is given by

$$
\frac{d Q_{k}}{d t}=\frac{1}{m_{k}} \frac{\operatorname{Im}\left(\psi^{*} \nabla_{k} \psi\right)}{\psi^{*} \psi}
$$

evaluated at the current configuration $Q$, where $m_{k}$ is the mass of particle $k$.

While this dynamics is local in configuration space, local in configuration space is not local in spacetime. Indeed, since a single point in configuration space represents the simultaneous positions of every particle, one must choose a preferred inertial frame in order to have a configuration space representation at all. Moreover, according to Bohm's dynamics, the velocity of a particle at a time is typically a function of the simultaneous positions of distant particles. And since it is this feature of the dynamics that explains the correlated results of EPR experiments in Bohm's theory, one might naturally conclude that the Bohmian dynamics is essentially incompatible with relativity.

Since both the evolution of the wave function and the evolution of the particle configuration are fully deterministic in Bohmian mechanics, in order to get the standard quantum probabilities, one must assume a special statistical boundary condition. The distribution postulate requires that there be a time $t_{0}$ where the epistemic probability density for the configuration $Q$ is given by $\rho\left(Q, t_{0}\right)=\left|\psi\left(Q, t_{0}\right)\right|^{2}$. If the distribution postulate is satisfied, 
one can show that Bohm's theory makes the standard quantum statistical predictions as epistemic probabilities for possible particle configurations.

Basic Bohmian mechanics can be generalized to make virtually any discrete physical quantity determinate. In particular, generalized Bohmian mechanics can be used to provide a hidden-variable theory where field quantities, rather than particle positions, are always determinate. Just as in the basic version of the theory, the standard quantum-mechanical state $\psi$ evolves in the usual unitary deterministic way and an auxiliary dynamics describes the time-evolution of the determinate physical quantity. The only significant difference is that the auxiliary dynamics is typically stochastic.

There are several empirically equivalent ways to specify an auxiliary dynamics for generalized Bohmian mechanics. The following describes the choices made by Bell (1984) and Vink (1993). Suppose that the current value of discrete physical quantity $Q$ is $q_{m}$. The probability that the value jumps to $q_{n}$ in the time interval $d t$ is $T_{m n} d t$, where $T_{m n}$ is an element in a transition matrix that is completely determined by the evolution of the wave function. More specifically, the wave function evolves according to the time-dependent Schrödinger equation

$$
i \hbar \partial_{t}|\psi(t)\rangle=H|\psi(t)\rangle,
$$

where $H$ is the global Hamiltonian. The probability density $P_{n}$ is defined by

$$
P_{n}(t)=\left|\left\langle q_{n} \mid \psi(t)\right\rangle\right|^{2}
$$

and the source matrix $J_{m n}$ is defined by

$$
J_{m n}=2 \operatorname{Im}\left(\left\langle\psi(t) \mid q_{n}\right\rangle\left\langle q_{n}|H| q_{m}\right\rangle\left\langle q_{m} \mid \psi(t)\right\rangle\right) .
$$

Finally, if $J_{m n} \geq 0$, then for $n \neq m$

$$
T_{m n}=J_{m n} / \hbar P_{m} ;
$$

and if $J_{m n}<0$, then $T_{m n}=0$. On analogy with the Bohmian particle dynamics, here one can think of the change in the discrete value of $Q$ as a discrete random-walk in $Q$-space biased by a compressible fluid with current $J_{m n}$. If the distribution postulate is satisfied here, this dynamics makes the standard quantum statistical predictions for the value of the determinate quantity $Q$ (Vink 1993). 
That both the basic and generalized formulations of Bohmian mechanics make the same empirical predictions as standard quantum mechanics for whatever physical quantity one takes as determinate has two immediate consequences. While the dynamics is clearly incompatible with relativity in the sense that one must choose a preferred inertial frame, if the distribution postulate is satisfied, then (i) one cannot communicate superluminal signals in either theory and (ii) there is no empirical way to detect which preferred inertial frame was used to calculate the evolutions of the determinate physical quantities. There is a sense then in which Bohmian mechanics is observationally compatible with relativity.[4] But one can get a stronger sort of compatibility between the two theories by sacrificing the dynamical explanations Bohm's theory typically provides.

A hidden-variable spacetime map consists in an assignment of a local value of each hidden variable to each region of spacetime. Both the basic and generalized formulations of Bohmian mechanics provide frame-dependent procedures for constructing such spacetime maps.

The basic formulation of Bohmian mechanics allows one to construct particle-trajectory spacetime maps. Choose a preferred inertial frame. Use the associated family of spacelike hyperplanes of simultaneity to define a $3 \mathrm{~N}$ dimensional configuration space associated with the preferred frame. Choose an appropriate wave function in configuration space at an initial proper time. Subject to the probabilities specified by the distribution postulate, randomly choose an initial particle configuration. Run the standard non-relativistic unitary dynamics on the wave function in configuration space associated with the preferred inertial frame and run the auxiliary dynamics on the determinate configuration (using the proper time for the preferred frame in each case). Then map the individual particle trajectories represented by the evolution of the preferred configuration to worldlines in Minkowski spacetime.

A closely related procedure can be used to construct the set of all possible Bohmian particle-trajectory spacetime maps together with a prior probability distribution over the set. Start with the wave function in the configuration space associated with the preferred inertial frame at an initial time. Suppose that an initial particle configuration has been determined subject to the distribution postulate, but that this is all one knows about the initial configuration. The distribution postulate then determines an epistemic probability distribution over possible configurations at a time in the preferred frame. Rather than tracking the evolution of the actual configuration, which 
by assumption one does not know here, track the evolution of every possible configuration that is compatible with the initial epistemic probability distribution in configuration space as specified by the distribution postulate. This initial probability distribution might be understood as providing the prior epistemic probabilities for each possible type of particle-trajectory spacetime map in fact describing our physical world (each possible type of spacetime map since in basic Bohmian mechanics the initial probability distribution will be represented by a continuous probability density). These prior probabilities may then be updated by conditioning on what one learns from empirical experience concerning the determinate features of the actual spacetime trajectory map.

Here the physical theory is just a set of possible spacetime maps and a distribution over these maps representing the probability that a particular type of map correctly describes our physical world. What is usually thought of as the dynamics, the standard unitary dynamics and the Bohmian auxiliary dynamics, is here just a prescription for how to construct the set of possible spacetime maps. But perhaps it is natural in statistical theories like Bohmian mechanics to think of the theory as just providing a set of possibilities and a probability measure over the set. In any case, the theory consturcted here is just a spacetime version of the many-threads theory discussed in Barrett 1999 where the connection rule is replaced with the prescription for constructing a spacetime hidden-variable map. In other words, the present frame-dependent theory fits nicely with hidden-variable formulations of quantum mechanics generally.

As in basic Bohmian mechanics, the epistemic probabilities one gets by conditioning on new evidence will agree with the standard quantum probabilities particle trajectories. And both the actual particle-trajectory spacetime map and the epistemic probabilities over all possible such maps are perfectly compatible with relativity in the sense that each map is just a description of the local value of each determinate physical parameter (here particle position) in each region of Minkowski spacetime and the probabilities are just representations of our uncertainty concerning which spacetime map in fact correctly describes our world.[5] 


\section{$3 \quad$ Virtues and Vices}

The particle-trajectory spacetime maps produced by frame-dependent constructions in basic Bohmian mechanics certainly fall short of what one should want from a satisfactory hidden-variable spacetime map in relativistic quantum mechanics. While such maps are in some sense perfectly compatible with relativity, the phenomena they represent are manifestly non-relativistic. Such maps do not, for example, account for even elementary relativistic phenomena such as particle creation. But getting the right empirical predictions for such relativistic phenomena here may be more a matter of ingenuity rather than of general principle.

As a first step toward getting the right relativistic predictions, there is good reason to exchange particle-trajectory maps for field-value maps. Here generalized Bohmian mechanics might then be used to provide constructions of possible determinate-field spacetime maps. While there would be a matter of fact concerning which determinate-field spacetime maps accurately describe our world, one would not know which, so one would update the prior epistemic probability distribution over possible determinate-field maps (as given by the distribution postulate) by conditioning on empirical evidence concerning the actual spacetime field map of our world.

While this is a step in the right direction, in order to get the right relativistic phenomena one would also need to use an appropriate field-theoretic version of the unitary dynamics in the frame-dependent construction. Indeed, assuming that the distribution postulate gives the right epistemic probabilities, getting the right relativistic phenomena here would ultimately depend on having the right frame-dependent field dynamics. Providing an adequate hidden-variable dynamics for the such frame-dependent constructions would, insofar as there are regularities in our determinate measurement records, there are such rules for constructing possible hidden-variable spacetime maps.[6]

While such hidden-variable spacetime maps would exhibit unexplained, nonlocal correlations between the values of the determinate measurement records, there is a sense in which maps themselves would be perfectly compatible with relativity. Indeed, any empirically adequate formulation of relativistic quantum mechanics that allows one to represent determinate measurement records in spacetime at all would be associated with just such a determinate record spacetime map. So, if one does not like the spacetime 
maps exhibiting nonlocal correlations between measurement records that are associated with the present theory, it must be that one does not like how they are constructed. But here, unlike the the teleological spacetime maps considered earlier, the problem is not that frame-dependent maps are straightforwardly ad hoc. They are, after all, constructed using rules that may or may not yield the right empirical predictions. Rather, the problem here is that the dynamical laws that are adapted from the nonrelativistic hidden-variable theories are in the relativistic hidden-variable theory not really behaving as dynamical laws. Indeed, the frame-dependent rule for constructing hiddenvariable spacetime maps here is just part of the characterization of the set of possible spacetime maps.

Philosophers of physics noted that one can get a spacetime formulation of virtually any physical theory and that this formulation can be made covariant in precisely the sense in which general relativity is uncontroversially covariant. Michael Friedman provides just such a reminder in his discussion of the covariance and invariance of spacetime theories (1983, 46-70). He also provides a concrete example of how to construct a spacetime formulation of Newtonian mechanics that is covariant in the sense of general relativity (1983, 71-95). The same can be done for Bohmian mechanics. Indeed, it is the ease of producing such covariant formulations of most any physical theory that is typically taken to show why the possibility of a covariant formulation of a particular theory is a poor indicator of the degree of compatibility between the theory and relativity. Nevertheless, if such constructions can be made empirically adequate, they are clearly preferable to teleological spacetime maps constructed from a collection of actual measurement results that guarantees empirical adequacy by stipulation.

The frame-dependent rules do not provide dynamical, mechanical, or causal explanations for the determinate events represented in the constructed spacetime maps. But, while neither dynamical, mechanical, nor causal, these rules nevertheless code for empirical regularities in a way that provides for empirical predictions and exposes them to potential empirical refutation and they arguably allow one to support counterfactual conditionals as well as any spacetime theory does.

How comfortable one is with frame-dependent constructions ultimately depends on the sort of explanations one wants from relativistic quantum mechanics. By opting for frame-dependent rules that do nothing beyond characterizing the set of possible hidden-variable spacetime maps, one is left 
without the richer mechanical or causal explanations we like to have whenever we can get them. On the other hand, it is not at all clear whether we will always be able to get satisfactory mechanical or causal explanations. And the assumption that we will is particularly suspect in the context of our best spacetime theories and in the context of quantum mechanics and hiddenvariable formulations of quantum mechanics in particular.

Depending on what one requires from mechanical explanations, both Bell's theorem and the Kochen-Specker theorem may amount to no-go theorems for such explanations in any empirically adequate formulation of quantum mechanics. [7] Whether causal explanations of events are always possible in quantum mechanics also depends on what counts as a causal explanation. But if one must be able to support counterfactual conditionals in order to provide causal explanations, then we may end up not being able to give causal explanations in our best hidden-variable formulations of quantum mechanics.

Michael Dickson has argued that it is at least contentious to claim that Bohmian mechanics predicts nonlocal causal relations since it does not support the counterfactual conditionals one might require in order to make sense of causal relations at all $(1998,202-8)$. More specifically, in the context of basic Bohmian mechanics, one can only support counterfactual conditionals of the sort represented by "What would have happened if the experiment had been set up differently?" by supposing that at least some particles were distributed differently than stipulated by the distribution postulate. But since it is the initial state being selected as stipulated by the distribution postulate that explains quantum probabilities in Bohmian mechanics, the theory cannot support such the counterfactual conditionals.

One problem with this argument is that the distribution postulate does not stipulate any particular initial state. Rather, it just requires that an initial state be randomly selected subject to the standard quantum probabilities. One might defend Dickson's conclusion by insisting that the particular initial state required for the analysis of a particular counterfactual conditional is never a state randomly selected subject to the distribution postulate. But there is another line of argument available.

The distribution postulate must be satisfied in order to get the right quantum statistical predictions given the standard Bohmian dynamics. But one can get essentially the same statistical predictions with a weaker statistical boundary condition if one adds an appropriate stochastic term to the auxiliary dynamics. That is, there is a trade-off between the dynamics and the 
statistical boundary condition in hidden-variable formulations of quantum mechanics like Bohmian mechanics such that one can get the right statistical predictions with many different dynamical-law-boundary-condition pairs. This is closely tied to the fact that there is typically no empirical way to determine what the actual particle dynamics is in a world described by even basic Bohmian mechanics. For our purposes, the important point here is that in a hidden-variable theory like Bohmian mechanics the boundary conditions and the dynamical laws work together to yield the right statistical consequences. The associated moral is that there can be little epistemic or methodological justification for arguing that boundary conditions and dynamical laws have an essentially different status in Bohmian mechanics: it is only together that they explain the standard quantum statistics.

If this is right, if the boundary conditions have the same status as the dynamical evolution of the state in Bohmian mechanics, then one cannot analyze causal relations by considering counterfactual boundary conditions. That is, if I am right here, to support a counterfactual conditional by considering a different boundary condition in Bohmian mechanics would be akin to supporting a counterfactual conditional by considering different dynamical laws. And counterfactual conditionals supported by considering counterfactual dynamical laws would clearly tell us nothing about the causal structure of our actual world. Similarly, if there is no principled difference in the methodological status of boundary conditions and dynamical laws, one should not worry much about replacing dynamics laws by the boundary conditions represented by the frame-dependent rules used to characterize possible spacetime maps.

The intimate relationship between dynamical laws and boundary conditions that prevents the analysis of causal structure by considering counterfactual boundary conditions is not limited to hidden-variable formulations of quantum mechanics. In general relativity, one might think of the geometry of spacetime and the distribution of matter as mutually constraining. The geometry and the matter distribution either satisfy the field equations together and thus represent a physically possible spacetime and matter distribution or they do not satisfy the field equations and thus do not represent a physically possible spacetime and matter distribution. Moreover, in general relativity, just as with frame-dependent hidden-variable spacetime maps, local spacetime stucture typically does not determine global spacetime stucture. Since the field equations of general relativity represent a global constraint on the 
relationship between the distribution of matter and the geometry of spacetime, if one tries to analyze causal structure by asking about a counterfactual distribution of matter in some spacetime region, the answer must typically be that the theory simply cannot support such a counterfactual.

That hidden-variable theories like Bohmian mechanics and that spacetime theories like general relativity do not generally support analyses of dynamical, mechanical, or causal structure that depend on the straightforward evaluation of counterfactual conditionals, is certainly relevant to how much we should worry that hidden-variable spacetime maps constructed from framedependent rules do not provide dynamical, mechanical, or causal explanations.

\section{Conclusion}

Frame-dependent hidden-variable constructions of spacetime maps are clearly preferable to teleological constructions since the former at least explains why the statistical properties of the observed measurement records are to be expected while the latter take the observed measurement records as given then simply stipulate an appropriate corresponding spacetime map. There are explanatory costs to settling for frame-dependent rules for characterizing possible spacetime maps and an epistemic probability distribution over the set. In particular, this sort of relativisitic hidden-variable formulation of quantum mechanics cannot provide the dynamical, mechanical, or causal explanations we like to have whenever we can. On the other hand, it is difficult to find a formulation of quantum mechanics where one can provide an empirically adequate dynamical explanation of one's determinate measurement records that is also compatible with relativity. And there are independent reasons to suppose that traditional sorts of mechanical and causal explanations may not ultimately be possible in hidden-variable formulations of quantum mechanics or our best spacetime theories anyway. 


\section{ENDNOTES}

1. I would like to thank David Malament and Brian Woodcock for helpful discussions and comments.

2. His discussion here is built on the earlier work of Aharonov, Bergmann, and Lebowitz and directly inspired the later work of Hellwig and Kraus (1970). It also foreshadowed relativistic collapse proposals like Aharonov and Albert's (1983) and hyperplane-dependent collapse theories.

3. An approach along these lines was suggested by Schlieder (1968). See Hellwig and Kraus $(1970,569)$ for a description of how Schlieder's spacetime maps are updated.

4. Peter Holland has argued that while some sort of violation of relativistic intuitions is "inevitable in a theory whose basic dynamical equations are defined in configuration space rather than ordinary spacetime," in Bohmian mechanics, relativity is nevertheless "statistically valid" $(1993,498)$. This sort of compatibility between Bohmian mechanics and relativity has often been noted. See for example, Albert (1992) and (2000), Bohm and Hiley (1993), Maudlin (1994) and (1996), and Dickson (1998).

5. That such relativistic particle theories are possible shows the sense in which particle no-go theorems like Malament's (1996) are contingent on exactly how one seeks to explain determinate measurement records in relativistic quantum mechanics. See Dickson (1998, 214-5) and Barrett (2002) for further discussions of this point.

6. Explanations of relativistic phenomena will ultimately turn on the specific choice of the frame-dependent dynamics. Ideally, one would want these explanations to be the result of some special novelty generated by the interaction between the basic principles of quantum mechanics and those of relativity, but it is unclear to me exactly how this could work in a framedependent construction. If this cannot be accomplished, it would represent 
an important sense in which such constructions are ad hoc.

7. These theorems suggest that one may not be able to get all of the traditional ingredients for mechanical explanations in quantum mechanics (a full set of determinate quantities, locally mediated interactions, functional relationships between quantities, etc.). See Bell (1987), Kochen and Specker (1967), and Bub (1997) for descriptions and discussions of these theorems. 


\section{REFERENCES}

Aharonov, Y. and D. Z Albert: 1981, 'Can We Make Sense out of the Measurement Process in Relativistic Quantum Mechanics?' Physical Review D 24(2): 359-70.

Albert, D. Z: 1999, 'Special Relativity as an Open Question.' In H. Breuer and F. Petruccione (eds.) State Vector Reduction in Relativistic Quantum Theory: Proceedings of the Workshop held at the instituto Italiano per gli Studi Filosofici, Naples, April 3-4 1998. (1-30) Berlin: Springer Verlag.

1992, Quantum Mechanics and Experience, Harvard University Press, Cambridge.

Barrett, J. A.: 2003 'Are Our Best Physical Theories Probably and/or Approximately True?' Forthcoming in Philosophy of Science.

2002 'On the Nature of Measurement Records in Relativistic Quantum Field Theory' in Ontological Aspects of Quantum Field Theory, M. Kuhlmann, H. Lyre, and A. Wayne (eds), New Jersey: World Scientific, 165-79.

2000 'The Persistence of Memory: Surreal Trajectories in Bohm's Theory' Philosophy of Science 67(4):680-703, 2000 Dec.

1999, The Quantum Mechanics of Minds and Worlds, Oxford: Oxford University Press.

Bell, J. S.: 1987, Speakable and Unspeakable in Quantum Theory, Cambridge University Press, Cambridge.

1984, 'Beables for Quantum Field Theory', CERN-TH.4035/84. Reprinted in Bell (1987, 173-80). 
1982, 'On the Impossible Pilot Wave,' Foundations of Physics 12:989-899. Reprinted in Bell (1987,159-168).

1981, 'Quantum Mechanics for Cosmologists,' in Quantum Gravity 2, C. Isham, R. Penrose, and D. Sciama (eds.), Oxford: Clarendon Press, 611-637. Reprinted in Bell (1987,117-138).

Bloch, I.: 1967, 'Some Relativistic Oddities in the Quantum Theory of Observation,' Physical Review 156(5):1377-84.

Bohm, D.: 1952, 'A Suggested Interpretation of Quantum Theory in Terms of "Hidden Variables", ' Parts I and II, Physical Review 85, 166-179, 180-193.

Bohm, D. and B. J. Hiley: 1993, The Undivided Universe: An Ontological Interpretation of Quantum Theory. London: Routledge.

Bub, J.: 1997, Interpreting the Quantum World, Cambridge: Cambridge University Press.

Dickson, M.: 1998, Quantum Chance and Nonlocality. Cambridge: Cambridge University Press.

Friedman, M.: 1983, Foundations of Spacetime Theories. Princeton: Princeton University Press.

Hellwig, K.E. and K. Kraus: 1970, "Formal Description of Measurements in Local Quantum Field Theory," Physical Review D 1(2):566-71.

Holland, P.: 1993, The Quantum Theory of Motion. Cambridge: Cambridge University Press.

Kochen, S. and E. P. Specker: 1967, 'The Problem of Hidden Variables in Quantum Mechanics', Journal of Mathematics and Mechanics 17:59-87.

Malament, D.: 1996, 'In Defense of Dogma: Why There Cannot be a Relativistic Quantum Mechanics of (Localizable) Particles', In R. Clifton (ed.), Perspectives on Quantum Reality, Dordrecht: Kluwer Academic Publishers, 
$1-10$.

Maudlin, T.: 1994, Quantum Nonlocality and Relativity, Oxford: Blackwell.

1996, 'Spacetime in the Quantum World' in J. T. Cushing, A. Fine and S. Goldstein (eds) Bohmian Mechanics and Quantum Theory: An Appraisal Netherlands: Kluwer Academic Publishers, pp. 285-307.

Schlieder, S.: 1968, 'Einige Bemerkungen zur Zustandsänderung von relativistischen quantenmechanischen Systemen durch Messungen und zur Lokalitätsforderung' Communications of Mathematical Physics 7:305-31.

Vink, J. C.: 1993, 'Quantum Mechanics in Terms of Discrete Beables,' Physical Review A 48:1808-1818. 